\title{
Skewness as measure of the invariance of instantaneous renormalized drop diameter distributions - Part 1: Convective vs. stratiform precipitation
}

\author{
M. Ignaccolo and C. De Michele \\ DIIAR, Politecnico di Milano, Milano, Italy \\ Correspondence to: M. Ignaccolo (m.ignaccolo@diiar.polimi.it) \\ Received: 26 May 2011 - Published in Hydrol. Earth Syst. Sci. Discuss.: 14 June 2011 \\ Revised: 16 December 2011 - Accepted: 19 December 2011 - Published: 3 February 2012
}

\begin{abstract}
We investigate the variability of the shape of the renormalized drop diameter instantaneous distribution using of the third order central moment: the skewness. Disdrometer data, collected at Darwin Australia, are considered either as whole or as divided in convective and stratiform precipitation intervals. We show that in all cases the distribution of the skewness is strongly peaked around 0.64. This allows to identify a most common distribution of renormalized drop diameters and two main variations, one with larger and one with smaller skewness. The distributions shapes are independent from the stratiform vs. convective classification.
\end{abstract}

\section{Introduction}

The term drop size distribution (DSD) acknowledges the stochastic nature of the occurrence of drop diameters. In particular the DSD is the concentration $N(D)$ of drops per unit volume and unit diameter, namely

$N(D)=N_{V} p(D)$

where $N_{V}$ is the number of drops per cubic meter and $p(D)$ is the density per diameter millimeter. Marshall and Palmer (1948) used an exponential functional form for the density $p(D)$. However it was soon clear that the exponential form was a result of the long (as long as $30 \mathrm{~min}$ ) time intervals used for sampling drop diameters. The shape of DSDs is highly variable even inside a single shower, a property which has led Joss and Gori (1978) to introduce the concept of instantaneous DSDs (distributions sampled over 1-2 min time intervals). Ulbrich (1983) introduced the gamma distribution as functional form for fitting the instantaneous DSDs. Although other functional forms have been proposed (Feingold and Levin, 1987), the gamma distribution is the one adopted in the overwhelming majority of cases.

Aside from the search of a proper functional form to best describe the DSD, investigations have been made to link the high variability of DSDs to few parameters following the introduction of "renormalized" DSDs by Sekhon and Srivastava (1971). The goal of this type of analysis is to describe DSDs in terms of rainfall bulk variables (e.g. rainfall rate, liquid water content, mean volume diameter) and/or identify a possible "universal", invariant in space and time, shape for the renormalized DSD. So far many different renormalization procedures have been proposed (Willis, 1984; Sempere Torres et al., 1994; Maki et al., 2001; Testud et al., 2001; Uijlenhoet et al., 2003; Campos et al., 2006; Hazenberg et al., 2011). The results are not conclusive in the sense that an "universal" shape as not been identified, yet the existing evidences indicate that indeed the great variability observed in the distributions of drop sizes could be described with few parameters.

A novel renormalization procedure has been introduced recently by Ignaccolo et al. (2009). The renormalized spectra have shown (Ignaccolo and De Michele, 2010) to posses the following properties. (1) Synoptic origin invariance for a fixed observation site (Darwin, AUS): convective and stratiform precipitation databases have same distribution. (2) Rainfall rate invariance for a fixed observation site (Darwin, AUS): databases built according to different rainfall rate classes (Tokay and Short, 1996) share a common distribution. (3) Cross invariance: the distributions in (1) and (2) are essentially identical. 
Do these results indicate the possible existence of a "universal" drop diameters distribution? In this paper we investigate this matter. More in particular we want to discuss the possible invariance of instantaneous, 1 min sampling, renormalized drop spectra. In fact, the properties (1), (2), and (3), above mentioned, refer to "averaged" renormalized spectra (see Sect. 2 for details). Are these results just due to "averaging" or they reflect an intrinsic dynamical property of rainfall? If the instantaneous renormalized spectra are all equal (strong equality $\rightarrow$ existence of a universal distribution) the invariance of "averaged" renormalized spectra (Ignaccolo et al., 2009) and (Ignaccolo and De Michele, 2010) is a trivial consequence of the existence of a "universal" distribution. If the instantaneous renormalized spectra manifest a high degree of variability not reducible to a definite criterion (weak/no equality) the invariant properties reported in Ignaccolo et al. (2009) and Ignaccolo and De Michele (2010) are merely an accident. A third possibility is the occurrence of a case in between these two extremes: moderate invariance.

To answer these questions, we investigate the skewness $\gamma$ of instantaneous drop size distributions in addition to the two renormalization parameters adopted in Ignaccolo et al. (2009), the mean, $\mu$, and the standard deviation, $\sigma$, of the drop diameter. In the case of strong equality the skewness of renormalized instantaneous spectra is a fixed value, while in the case of weak/no equality one expects a flat distribution of skewness value. The work is organized as follows. Section 2 illustrates the methodology adopted for our analysis, while Sect. 3 illustrates how data are processed prior to analysis. We present our results in Sect. 4 and then draw our conclusion, Sect. 5.

\section{Methodology}

\subsection{Renormalization}

Given a renormalization time interval $I$, of length typically in the range $1-5 \mathrm{~min}$, the renormalization procedures usually adopted in Literature (Willis, 1984; Sempere Torres et al., 1994; Maki et al., 2001; Testud et al., 2001; Uijlenhoet et al., 2003; Campos et al., 2006; Hazenberg et al., 2011) operate a rescaling of both the diameter $D$ and the instantaneous drop size distribution $\mathcal{N}_{I}(D)$, the DSD relative to the drops inside the renormalization time interval:

$$
\left\{\begin{array}{l}
\left(D, \mathcal{N}_{I}(D)\right) \rightarrow\left(D^{*}=D / X_{I}, \mathcal{N}_{I}^{*}\left(D^{*}\right)=\mathcal{N}_{I}(D) / Y_{I}\right) \\
\mathcal{N}_{I}(D)=\frac{N_{I}}{A T} \frac{p_{G, I}(D)}{v(D)}
\end{array}\right.
$$

The variables $X_{I}$ and $Y_{I}$ are instantaneous bulk variables (e.g. volume mean diameter and liquid water content respectively) or power functions of those bulk variables used to obtain the renormalized diameter $D^{*}$ and renormalized instantaneous spectra $\mathcal{N}_{I}^{*}\left(D^{*}\right)$. The existence of an invariant spectrum would result in a "single" shape for the graph
$\left(D^{*}, \mathcal{N}_{I}^{*}(D)\right)$. The bottom equality of Eq. (2) is the relationship between the instantaneous DSD and the instantaneous probability density of diameter observed at the ground $p_{G, I}(D)$. The parameter $A$ is the base area, in $\mathrm{m}^{2}$, of the unit volume where $\mathcal{N}_{I}(D)$ is the concentration (for disdrometer data $A$ is the catchment area of the instrument), $T$ is the length of the renormalization time interval in seconds, while $N_{I}$ is the total drop count during the time interval $T$. Finally, $v(D)$ is the falling speed, in $\mathrm{m} \mathrm{s}^{-1}$, of a drop of diameter $D$.

The renormalization procedure proposed by Ignaccolo et al. (2009) and Ignaccolo and De Michele (2010) operates as follows. For each renormalization time interval $I$ :

$$
\left\{\begin{array}{l}
D \rightarrow D_{R}=\frac{D-\mu_{I}}{\sigma_{I}} \\
p_{G, I}(D) \rightarrow p_{G, I}\left(D_{R}\right)=\sigma_{I} p_{G, I}\left(\sigma_{I} D_{R}+\mu_{I}\right)
\end{array}\right.
$$

where $\mu_{I}$ and $\sigma_{I}$ are the mean, and standard deviation of the drop diameter observed at the ground. This renormalization procedure operates a change of variable from the diameter $D$ to the renormalized diameter $D_{R}$ having zero mean and unit variance. The instantaneous renormalized spectrum is in this case the instantaneous probability density $p_{G, I}\left(D_{R}\right)$ of the renormalized drop diameter. This density is obtained from that of drop diameter observed at the ground $p_{G, I}(D)$ using the second identity of Eq. (3). The rationale behind this choice of Eq. (3) is that the time series $\left\{D_{k}\right\}, k=1,2,3, \ldots$, of drop diameters is derived from a stochastic process which would be stationary if it were not for a variable mean and a variable standard deviation (e.g. the works of Kostinski and Jameson, 1997 and Smith, 1993 support this ansatz). If so the renormalization procedure removes the non-stationarity, so that sequences of renormalized diameters relative to two different datasets should have the same probability density $p_{G}\left(D_{R}\right)$. This is precisely what is observed in Ignaccolo et al. (2009) and Ignaccolo and De Michele (2010).

Disdrometers categorize drops in diameter classes. A drop in the $j$-th class has to be considered as the occurrence of a drop with a random diameter value $D$ uniformly distributed in the range $\left[D_{j}-\Delta_{j} / 2, D_{j}+\Delta_{j} / 2\right]$, where $D_{j}$ and $\Delta_{j}$ are the central value and the width of the class. We refer to this inaccuracy as the quantization error. Using the first relationship of Eq. (3), the renormalized drop diameter $D_{R}$ associated to a drop in the $j$-th disdrometer class can be any number, with equal probability, in the inter$\operatorname{val}\left[\left(D_{j}-\Delta_{j} / 2-\mu_{I}\right) / \sigma_{I},\left(D_{j}+\Delta_{j} / 2+\mu_{I}\right) / \sigma_{I}\right]$. Another consequence of the quantization error is that the probability density $p_{G, I}(D)$ of drop diameter, as estimated from disdrometer drop counts, has to be considered as a step function, namely

$p_{G, I}(D)=\sum_{j} p_{G, I}^{j} \chi_{j}(D)=\sum_{j} \frac{n_{I, j}}{N_{I} \Delta_{j}} \chi_{j}(D)$

where the value of the indicator function $\chi_{j}(D)$ is 1 if $D$ is inside the boundary of the $j$-th diameter class, otherwise $\chi_{j}(D)=0$. The symbol $p_{G, I}^{j}$ indicates the value of $p_{G, I}(D)$ 
inside the $j$-th diameter class, which is the ratio between $n_{I, j}$, the drop count in the $j$-th diameter class, and the product between $\Delta_{j}$, the width of the $j$-th diameter class, and $N_{I}$, the total disdrometer count inside the renormalization time interval considered. The instantaneous renormalized spectra $p_{G, I}\left(D_{R}\right)$ is also a step function as it is obtained applying both relations of Eq. (3) to Eq. (4).

An advantage of the renormalization procedure of Eq. (3) with respect classical renormalizations, Eq. (2), is that one can associate to a data set of disdrometer counts a sequence of renormalized drop diameters, and thus define the corresponding renormalized spectra of the entire data set: $\pi_{G}\left(D_{R}\right)$. This association is done as follows. Inside each renormalization time interval a random number uniformly distributed in the interval $\left[\left(D_{j}-\Delta_{j} / 2-\mu_{I}\right) / \sigma_{I}\right.$, $\left.\left(D_{j}+\Delta_{j} / 2+\mu_{I}\right) / \sigma_{I}\right]$ is selected for each drop in the $j$-th diameter class. One problem with this procedure is that it is not unique, so that each repetition creates a new sequence of renormalized drop diameters and therefore a new probability density $\pi_{G}\left(D_{R}\right)$. A possibility is that of repeating the renormalization procedure $M$ times and define the probability density $p_{G}\left(D_{R}\right)$ of a particular dataset as the average density of the $M$ realizations:

$p_{G}\left(D_{R}\right)=\frac{1}{M} \sum_{k=1}^{M} \pi_{G}^{k}\left(D_{R}\right)$

where the symbol $\pi_{G}^{k}\left(D_{R}\right)$ is the renormalized spectra relative to the $k$-th renormalization procedure. Ignaccolo and De Michele (2010) show that already for $M=100$ one obtains a fairly stable average value and the fluctuations around the average are negligible down to a frequency of $\sim 100 / N$, with $N$ being the total number of drops in the dataset. Alternatively, the probability density of renormalized drop diameter obtained with a single run of the renormalization procedure significantly deviates from the average $p_{G}\left(D_{R}\right)$ obtained from many realizations only for frequency values $\lesssim 100 / N$. Hereby we will denote by $p_{G}\left(D_{R}\right)$ the average density for an infinite number of realizations of the renormalization procedure since an analytical formula can be derived for this quantity. Let us consider the $k$-th renormalization procedure. The number of drops in the infinitesimal interval $d D_{R}$ centered around the value $D_{R}$ is

$$
\begin{aligned}
N \pi_{G}^{k}\left(D_{R}\right) d D_{R} & =\sum_{I} N_{I} p_{G, I}\left(D_{R}\right) d D_{R} \\
& +\sum_{I} \epsilon_{I}^{k}\left(D_{R}\right) d D_{R}
\end{aligned}
$$

where the sums are made over all renormalizations time intervals. The first term of the right hand side of Eq. (6), not depending on the parameter $k$, is the expected value from the particular drop counts observed in the interval, $N_{I}$ is the total drop count in the interval and the probability density $p_{G, I}\left(D_{R}\right)$ is obtained from $p_{G, I}(D)$ using the second relation in Eq. (3). The second term of Eq. (6), depending on the parameter $k$, expresses the statistical fluctuations due to the quantization error of the disdrometers: a drop in the $j$-th diameter class is assigned a random value of the renormalized diameter in the range $\left[\left(D_{j}-\Delta_{j} / 2-\mu_{I}\right) / \sigma_{I}\right.$, $\left.\left(D_{j}+\Delta_{j} / 2+\mu_{I}\right) / \sigma_{I}\right]$. Note that $\int \epsilon_{I}^{k}\left(D_{R}\right) d D_{R}=0$ so that the integration with respect to $D_{R}$ of Eq. (6) simply returns the total number of drops in the database considered. Substituting Eq. (6) into Eq. (5), and adopting the limit $M \rightarrow \infty$ has the effect of removing the contributions of the statistical fluctuations, second term of the right hand size of Eq. (6), so that

$p_{G}\left(D_{R}\right)=\frac{1}{N} \sum_{I} N_{I} p_{G, I}\left(D_{R}\right)$.

\subsection{Calculation of renormalization parameters}

Given a renormalization time interval, the $n$-th instantaneous moment $M_{I, n}$ of the probability density $p_{G, I}(D)$ of drop diameter at the ground is

$M_{I, n} \underset{\text { def }}{=} \int_{0}^{+\infty} D^{n} p_{G, I}(D) d D$.

Since disdrometers classify drop diameters per class (quantization error) the probability density is a step function, Eq. (4), so that

$$
\begin{aligned}
M_{I, n} & =\sum_{j} p_{G, I}^{j} \int_{D_{j, L}}^{D_{j, R}} D^{n} d D=\sum_{j} \frac{n_{I, j}}{N_{I} \Delta_{j}} \\
& \times \frac{\left(D_{j, R}\right)^{n+1}-\left(D_{j, L}\right)^{n+1}}{n+1}
\end{aligned}
$$

where the sum is taken over the diameter classes, index $j$. The symbol $p_{G, I}^{j}$ indicates the value of $p_{G, I}(D)$ inside the $j$-th diameter class, while $n_{I, j}, \Delta_{j}, D_{j, L}$, and $D_{j, R}$ are respectively the drop count, width, left limit, and right limit of the $j$-th diameter class. Finally $N_{I}$ is the total disdrometer count inside the renormalization time interval considered. The renormalization procedure adopted here requires the calculation of two parameters: the mean $\mu_{I}$ and the standard deviation $\sigma_{I}$ of drop diameters. In addition to the renormalization parameters, the skewness $\gamma_{I}$ is evaluated and used as parameter to quantify the "equality" of instantaneous renormalized spectra. All these parameters can be obtained from the calculation of the $n$-th instantaneous moment $M_{I, n}$, Eq. (9), as follows

$$
\left\{\begin{array}{l}
\mu_{I}=M_{I, 1} \\
\sigma_{I}=\sqrt{M_{I, 2}-\left(M_{I, 1}\right)^{2}} \\
\gamma_{I}=\frac{M_{I, 3}+2\left(M_{I, 1}\right)^{3}-3 M_{I, 1} M_{I, 2}}{\left[M_{I, 2}-\left(M_{I, 1}\right)^{2}\right]^{3 / 2}}
\end{array} .\right.
$$




\subsection{Skewness as measure of invariance}

To "measure" the invariance of two different instantaneous renormalized spectra $p_{G, I}\left(D_{R}\right)$, one could consider a statistical test of equality between sample distributions, e.g. Kolmogorov-Smirnov. This approach is not feasible in our case. (1) If $M$ is the total number of renormalization intervals, one has to test $M(M-1) / 2$ couples of renormalization time intervals, in our case $M=6863 \Rightarrow 23546953$ couples. (2) Statistical tests of equality have no transitive property. If the couples of renormalization time intervals $\left(I_{1}\right.$, $\left.I_{2}\right)$ and $\left(I_{2}, I_{3}\right)$ pass the test, nothing can be implied for the couple $\left(I_{1}, I_{3}\right)$. Thus one cannot simply use the number of couples which have the same spectra as an indication of the existence of an invariant distribution. (3) Inadequacy of tests of equality between sample distributions. Let us suppose that there is a universal distribution so that for each renormalization time interval $p_{G, I}\left(D_{R}\right)=f\left(D_{R}\right)$, then the instantaneous spectra $p_{G, I}(D)$ are obtained from $f\left(D_{R}\right)$ inverting the relations in Eq. (3) with given values $\mu_{I}^{\text {th }}$ and $\sigma_{I}^{\text {th }}$. The superscript "th" indicates theoretical values. We cannot directly observe $p_{G, I}\left(D_{R}\right)$ but only $p_{G, I}(D)$, and the use Eq. (10) to have an estimate of $\mu_{I}^{\text {th }}$ and $\sigma_{I}^{\text {th }}$. Thus the possible statistical differences between the renormalized spectra of two renormalization time intervals are due to (A) sampling fluctuations, the number of drop counts being smaller than infinity; (B) errors in estimating the theoretical values $\mu_{I}^{\text {th }}$ and $\sigma_{I}^{\text {th }}$, which introduce consequently errors in $p_{G, I}\left(D_{R}\right)$. However, tests of equality between sample distributions take in account only (A) and not (B) as source of statistical differences. Therefore these tests are susceptible to failure even in the case when an universal distribution exists.

Due to these limitations, we follow an alternative approach and consider the skewness $\gamma_{I}$ as the parameter to characterize the equality of instantaneous renormalized drop spectra. The rationale for this choice are (1) the densities $p_{G, I}\left(D_{R}\right)$ have all zero mean and unit variance in virtue of the renormalization procedure. Thus, the skewness is the next standardized moment which can be used to describe the distribution. (2) The renormalization procedure, Eq. (3), preserves the skewness: the instantaneous density $p_{G, I}(D)$ and $p_{G, I}\left(D_{R}\right)$ have the same skewness. In fact

$$
\begin{aligned}
& \int_{0}^{+\infty}\left(\frac{D-\mu_{I}}{\sigma_{I}}\right)^{k} p_{G, I}(D) d D=\int_{-\frac{\mu_{I}}{\sigma_{I}}}^{+\infty} D_{R}^{k} \sigma_{I} \\
& p_{G, I}\left(\sigma_{I} D_{R}+\mu_{I}\right) d D_{R}=\int_{-\frac{\mu_{I}}{\sigma_{I}}}^{+\infty} D_{R}^{k} p_{G, I}\left(D_{R}\right) d D_{R}
\end{aligned}
$$

where $k$ is any real number. Thus in general the renormalization procedure of Eq. (3) preserves not only the skewness $(k=3)$, but all the standardized moments ( $k$ integer).

\section{Data processing}

We use Joss Waldvogel disdrometer data at $1 \mathrm{~min}$ time resolution recorded in Darwin, Australia $\left(12.45^{\circ} \mathrm{S}, 130.83^{\circ} \mathrm{E}\right.$, $2 \mathrm{~m}$ a.m.s.l.) for 97 consecutive days, from 4 November 2005 to 10 February 2006. This is the same database of Ignaccolo and De Michele (2010). Drop diameters are classified in 20 different classes covering the range $0.3-5.6 \mathrm{~mm}$. Moreover counts are corrected against the instrument dead time (Sauvageot and Lacaux, 1995). The total number of minutes in this dataset is 139680 of which only $26595(\simeq 19 \%)$ display at least one drop count. The total drop count is 2943435 . Reflectivity maps are available for the time intervals 9 November to 6 December 2005, and 6 January to 10 February 2006, allowing for stratiform versus convective classification through the identification of the bright band. A total of 19 stratiform and 33 convective time intervals were identified with this method (see the online material in Ignaccolo and De Michele, 2010). Parsing together all these intervals we obtain the stratiform subset (4669 min of which 4264 with a non null drop count for a total of 407277 drops) and the convective subset (2931 min of which 2267 with a non null drop count for a total of 1077488 drops).

We select the length of the renormalization time intervals $I$ to be $1 \mathrm{~min}$, the same as the time resolution of our dataset. One "artifact" affecting the renormalization procedure is the statistical errors in calculating the mean and standard deviation of the drop diameters for each renormalization time interval. To mitigate this issue, we exclude minutes with counts $\leq 60$, or with a number of occupied diameter classes $<3$ from the renormalization procedure. The rationale behind these threshold values is: (1) they identify a dynamical property of the rainfall phenomenon as the quiescent time intervals, intervals of sparse precipitation, with negligible contribution to the overall precipitated volume (we refer the reader to Ignaccolo et al., 2009 for a detailed discussion). (2) They allow a "reasonable" (law of large numbers) estimate of the mean and standard deviation of drop diameters. Once these minutes are removed, we are left with 6863 non-quiescent minutes and 2758320 drops for the Darwin database (1844 nonquiescent minutes and 355545 drops for the stratiform subset, and 1536 non-quiescent minutes and 1066299 drops for the convective subset).

Another possible source of artifacts is the outliers drop counts. With a choice of $1 \mathrm{~min}$ for the length of the renormalization time interval and 20 diameter classes the drop counts are mostly distributed in such a way to cover continuously all the diameter classes in between an initial and a final class: e.g. classes 4 to 16 have non zero counts and classes 1 to 3 and 17 to 20 are all empty. Occasionally there are class gaps, that is two non adjacent classes with non zero count are separated by one or more classes with null counts. These gaps are due to sampling fluctuations occurring for the classes with small probability of occurrence (if $N_{I}$ is the number of drops inside the renormalization time interval, as rule of 
thumb probabilities of the order of $1 / N_{I}-10 / N_{I}, N_{I} \gg 10$, are going to be affected by sampling fluctuations). Thus one can observe, e.g. a zero count for the classes 1 to 3 , non zero counts from class 4 to 7 , a count of 1 on class 8 followed by zeros from class 9 to 20 . To identify no gap regions containing the maximum count we proceed as follows. For each 1 min time interval with class gaps we seek the class with the maximum count, and then we move both to the left and right of this class until we either reach a zero count or the minimum (1) or maximum (20) diameter class of the instrument. We then calculate the fraction of the total number of drops inside the renormalization time interval belonging to the no gap region containing the maximum count. If the class gap is due to sampling fluctuations we expect this fraction to be large (e.g. $90 \%$ or larger). For the Darwin database 1091 of the $6863(\simeq 16 \%)$ renormalization time intervals have class gaps. For 86 intervals, out of the 1091 with gaps, the no gap region contains less than $90 \%$ of the total drop count.

Even if the drop counts following a gap constitute a small portion of total drop count inside a renormalization time interval, their effects amount to (1) larger values for the parameters $\mu_{I}, \sigma_{I}$, and $\gamma_{I}$ (2) fatter left and right tail for the instantaneous probability densities function at ground $p_{G, I}(D)$ and $p_{G, I}\left(D_{R}\right)$ and for the probability $p_{G}\left(D_{R}\right)$ relative to the entire data set. A full disclosure of these effects, and the rationale for improving statistical accuracy by discarding outliers drop counts is presented in the Appendix of the companion paper (Ignaccolo and De Michele, 2012). Here, we set to zero the outliers drop counts before applying the renormalization procedure. The total number of drops of the 6863 non-quiescent renormalization time intervals considered is then reduced from 2758320 to $2753796, \sim-0.16 \%$ (from 355545 to $354743, \sim-0.22 \%$, for the stratiform subset, and from 1066299 to $1064561, \sim-0.16 \%$, for the convective subset).

\section{Results}

Figure 1 shows the probability $\operatorname{Pr}\left(\gamma_{I}\right)$ of having a renormalized drop spectrum $p_{G, I}\left(D_{R}\right)$ with skewness $\gamma_{I}$ for the entire Darwin database and for the stratiform and convective subsets. We see how the distribution for the entire dataset is peaked around the skewness value 0.64 . The probability $\operatorname{Pr}\left(\gamma_{I}\right)$ of the stratiform (convective) subset is more peaked (flat) around a slightly larger (smaller) skewness value when compared with the probability relative to the entire database (solid line in Fig. 1). This figure also indicates the existence of a substantial degree of invariance which we quantify using the concept of skewness class. A renormalization time interval $I$ belongs to the skewness class $r$ if the relative difference of the corresponding skewness $\gamma_{I}$ with respect the most probable value 0.64 is within the percentage range $[(r-1 / 2) \times 100,(r+1 / 2) \times 100]$ : e.g. skewness class zero (s0) implies $\gamma_{I}$ in the range [0.32, 0.96], skewness class

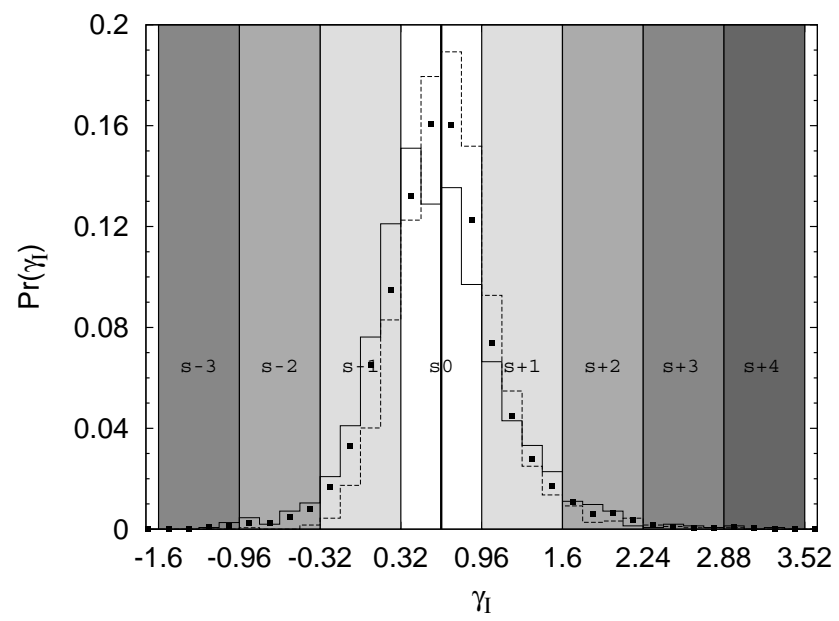

Fig. 1. The probability $\operatorname{Pr}\left(\gamma_{I}\right)$ for the entire (full squares), stratiform (dashed line), and convective (solid line) datasets at Darwin. The shaded regions indicate different skewness classes.

plus-one $(\mathrm{s}+1)$ implies $\gamma_{I}$ in the range $[0.96,1.60]$, while skewness class minus-two $(\mathrm{s}-2)$ implies $\gamma_{I}$ in the range $[-0.96,-0.32]$. Skewness classes are indicated in Fig. 1 with shadowed regions separated by vertical lines.

Table 1 reports for each database (all, stratiform, and convective) the percentages $I \%$ of the database number of renormalization time intervals belonging to a given skewness class, and the percentage $d \%$ of the database total number of drops belonging to renormalization time intervals in a given skewness class. We see how the percentage $I \%$ for the skewness class $\mathrm{s} 0$ is $\sim 64 \%$ for the stratiform database but $\sim 51 \%$ for the convective database. The other two skewness classes which are appreciably populated are $s+1$ and $s-1$, with $\mathrm{s}+1$ more predominant in the stratiform case and $\mathrm{s}-1$ more predominant in the convective case. However if we consider the percentage of $d \%$ of the total number of drops in the databases, we see a more balanced repartition among the skewness classes: $d \%$ is in the range $61-62 \%$ for skewness class $\mathrm{s} 0,14-18 \%$ for $\mathrm{s}-1$, and $16-19 \%$ for $\mathrm{s}+1$. The origin of this balance is depicted in Fig. 2 which illustrates the occupancy $O\left(N_{I}, \gamma_{I}\right)$, the number of couples $\left(N_{I}, \gamma_{I}\right)$ inside a given box in the $N_{I} \gamma_{I}$-plane. For the convective database higher values of the occupancy $O\left(N_{I}, \gamma_{I}\right)$ are observed for skewness class $\mathrm{s}+1$, however the drop count $N_{I}$ is not as high as for the skewness classes $\mathrm{s} 0$ and $\mathrm{s}-1$. Moreover, we notice how large $\left(N_{I}>1000\right)$ drop counts occur almost exclusively for skewness classes $\mathrm{s} 0, \mathrm{~s}+1$, and $\mathrm{s}-1$ : renormalized drop spectra with "extreme" skewness values are rare and coupled with relatively small drop counts.

Next, we divide the entire, stratiform and convective Darwin databases in subsets according to the skewness class of each renormalization time interval. We calculate the probability density $p_{G}\left(D_{R}\right)$ of the renormalized drop diameter $D_{R}$ for each subsets using Eq. (7). Figure 3 shows the results for 
Table 1. Classification of the databases (respectively all, stratiform, and convective) in classes of skewness using the number of time intervals $(I \%)$, and number of drops $(d \%)$.

\begin{tabular}{|c|c|c|c|c|c|c|}
\hline \multirow{2}{*}{$\begin{array}{l}\text { Database } \\
\text { Class of } \gamma\end{array}$} & \multicolumn{2}{|c|}{ All } & \multicolumn{2}{|c|}{ Stratiform } & \multicolumn{2}{|c|}{ Convective } \\
\hline & $I \%$ & $d \%$ & $I \%$ & $d \%$ & $I \%$ & $d \%$ \\
\hline$s-3[-0.96,-1.60]$ & 0.19 & 0.11 & 0 & 0 & 0.32 & 0.15 \\
\hline$s-2[-0.96,-0.32]$ & 1.74 & 1.14 & 0.21 & 0.08 & 2.40 & 1.99 \\
\hline$s-1[-0.32,0.32]$ & 20.99 & 18.61 & 14.48 & 14.12 & 25.91 & 18.33 \\
\hline s0 $[0.32,0.96]$ & 57.57 & 61.44 & 64.31 & 62.06 & 51.23 & 61.13 \\
\hline$s+1[0.96,1.60]$ & 16.37 & 15.87 & 18.6 & 19.11 & 16.53 & 17.33 \\
\hline$s+2[1.60,2.24]$ & 2.66 & 2.48 & 1.95 & 3.67 & 2.93 & 1.99 \\
\hline $\mathrm{s}+3[2.24,2.88]$ & 0.32 & 0.25 & 0.37 & 0.81 & 0.45 & 0.17 \\
\hline $\mathrm{s}+4[2.88,3.52]$ & 0.11 & 0.07 & 0.05 & 0.12 & 0.19 & 0.06 \\
\hline
\end{tabular}

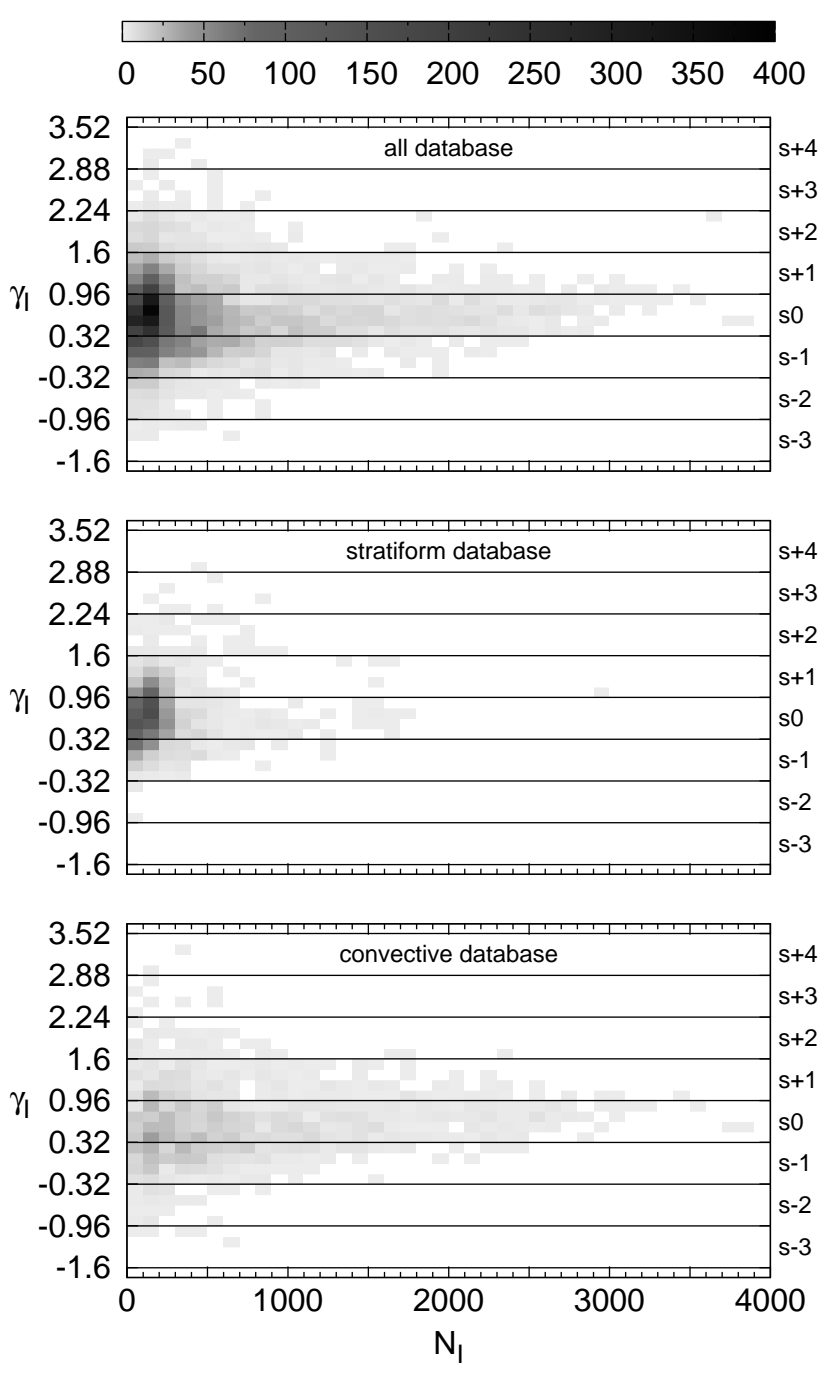

Fig. 2. The occupancy $O\left(N_{I}, \gamma_{I}\right)$ for the entire, stratiform, and convective datasets at Darwin. Horizontal lines denote the skewness classes. each skewness class in comparison with the density of the entire Darwin database. The classes $s+4$ and $s-3$ are not shown because of poor statistics: $s+4(s-3)$ has $8(13)$, $1(0)$, and 3 (5) renormalization time intervals in the entire, stratiform, and convective databases, respectively. For each skewness class, the probability density $p_{G}\left(D_{R}\right)$ does not depend on the particular dataset considered (entire, stratiform, or convective). The continuous line in all panels of Fig. 3 is the probability density $p_{G}\left(D_{R}\right)$ of the whole Darwin dataset (including all skewness classes). Comparing this density with the densities per skewness class, we see how the class $\mathrm{s} 0$ essentially defines the central part of the whole Darwin data set density, while the skewness classes of degree $r \gtrless 0$ largely influence of the tails of the distribution. The percentage $d \%$ of the database total number of drops belonging to renormalization time intervals in a given skewness class determines the shape of the probability density $p_{G}\left(D_{R}\right)$ of the entire dataset considered. From Table 1 we see that the variability of the percentage $d \%$ with respect to the databases considered is relatively small so that we expect a substantial invariance in distribution which is precisely what is observed in Ignaccolo and De Michele (2010).

The results depicted in Fig. 1 indicate that the probability density $p_{G}\left(D_{R}\right)$ relative to the skewness class $\mathrm{s} 0$ could be considered as a "standard" distribution $S\left(D_{R}\right)$ : the most probable distribution. Two main deviations from the most common distribution are observed, one with smaller $\left(p_{G}\left(D_{R}\right)\right.$ relative to the skewness class $\left.\mathrm{s}-1\right)$ and one with larger skewness $\left(p_{G}\left(D_{R}\right)\right.$ relative to the skewness class $\mathrm{s}+1)$.

\subsection{Sample variability of the skewness}

The limited drop count observed inside each renormalization time interval produces "errors" in the calculated value of the parameters $\mu_{I}, \sigma_{I}$, and $\gamma_{I}$. Even if there was an unique renormalized spectrum one can expect the observed values for the skewness inside a renormalization time interval to 

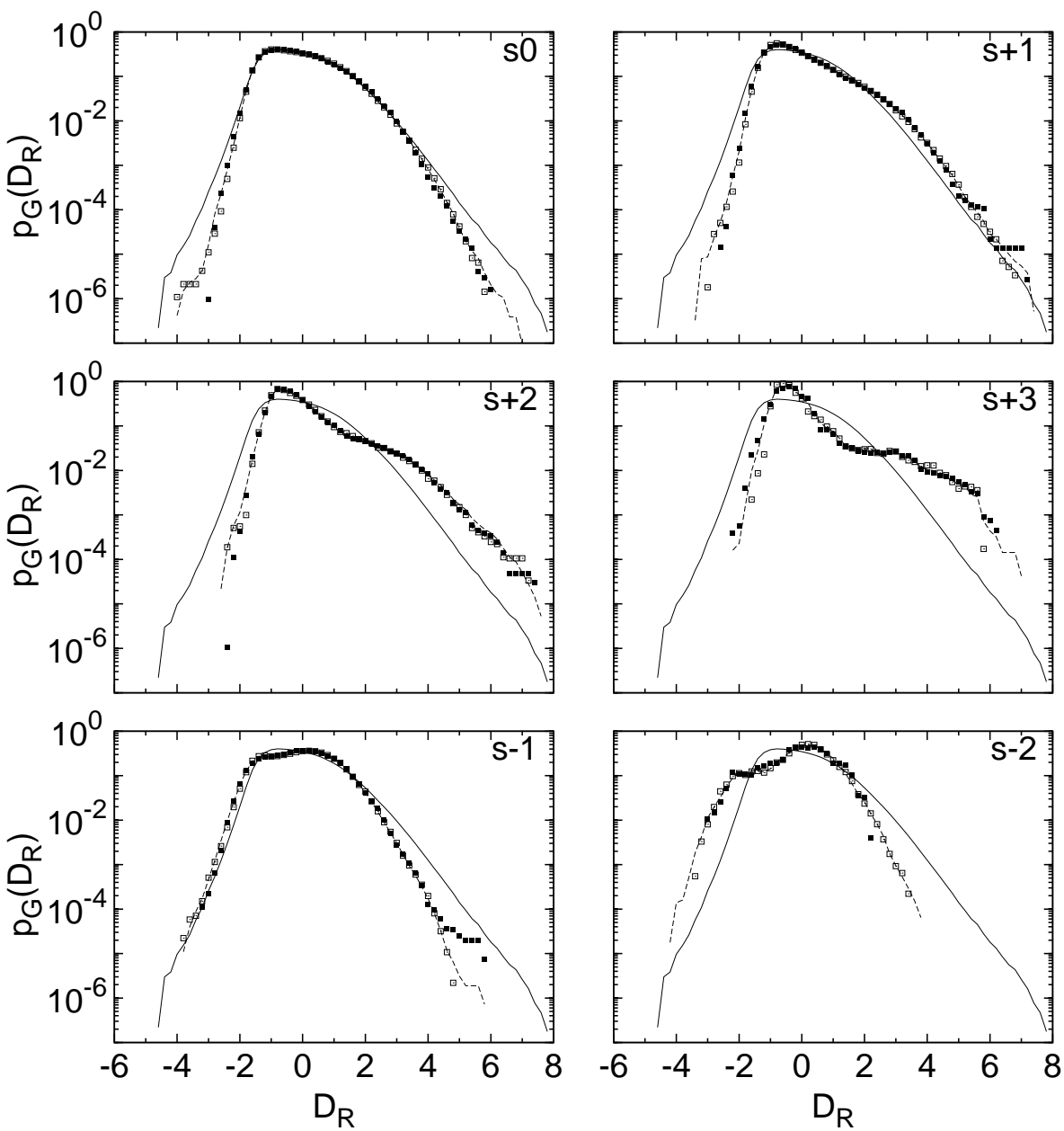

Fig. 3. The value of the probability density $P_{G}\left(D_{R}\right)$ for the skewness classes subsets obtained from the entire database (dashed line), the stratiform database (solid squares), and the convective database (open squares). The label on the top right corner of each panel indicates the skewness class: s0, s+1, s+2,s $+3, \mathrm{~s}-1, \mathrm{~s}-2$. The solid line denotes the probability density $P_{G}\left(D_{R}\right)$ for the entire Darwin database.

show some variability. Is thus possible that part of the deviation from the most probable value $\gamma_{I}=0.64$ shown in Fig. 1 might be due to sampling errors. To test this possibility, we use the standard distribution $S\left(D_{R}\right)$ to create an artificial sequence of drop counts which are supposed to have all the same skewness. For each renormalization time interval we extract $N_{I}$ renormalized diameters to obtain the simulated $(\operatorname{sim})$ instantaneous renormalized spectrum $p_{G, I}^{\mathrm{sim}}\left(D_{R}\right)$. Starting from $p_{G, I}^{\mathrm{sim}}\left(D_{R}\right)$, we use the mean $\mu_{I}$ and the standard deviation $\sigma_{I}$ of the renormalization time interval and invert the relationships of Eq. (3) to obtain the drop counts taking care of rejecting, if any occur, drops with a simulated diameter $D<0.3 \mathrm{~mm}$ since this is the minimum drop diameter detectable by a JW disdrometer.

We apply this procedure to the entire Darwin data set to obtain an artificial sequence. We then calculate the skewness values of the artificial sequence and compare its distribution with that observed in reality. Figure 4 shows the results. We see how the distribution of skewness values of the artificial sequence is much more peaked than the real one. For the artificial sequence, almost $92 \%$ of the renormalization time intervals are inside the skewness class s0, with $\sim 5 \%$ and $\sim 3 \%$ in the $\mathrm{s}-1$ and $\mathrm{s}+1$ skewness classes. Thus the observed spread of skewness values depicted in Fig. 1 can be ascribed to sampling fluctuation only partially (compare $5 \%$ and $3 \%$ with the values of $\% I$ in Table 1 ).

\subsection{Relationship among mean, standard deviation, and skewness}

Our statistical description associates to each renormalization time interval four parameters: the drop count $N_{I}$, the mean $\mu_{I}$, the standard deviation $\sigma_{I}$ and the skewness $\gamma_{I}$ of drop diameters. Only the mean and the standard deviation are used in the renormalization procedure of Eq. (3). More in general these four parameters are related to each other albeit in a statistical way. E.g. Ignaccolo and De Michele (2010) show that there is an approximate linear relation between the 


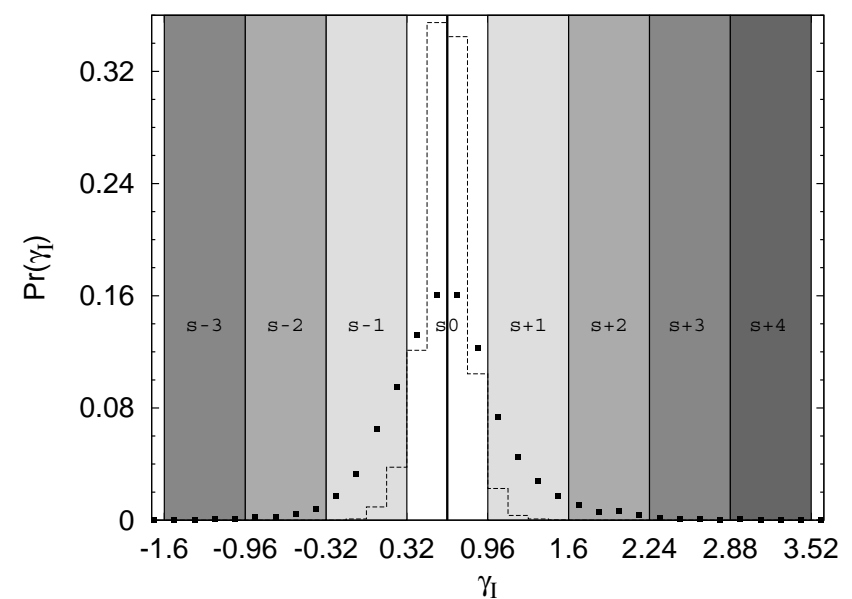

Fig. 4. The probability $\operatorname{Pr}\left(\gamma_{I}\right)$ for the entire dataset at Darwin (solid squares) and for an artificial realization (dashed line) created using the standard probability density $S\left(D_{R}\right)$. The shaded regions indicate different skewness classes.

average values of the parameters $\mu_{I}$ and $\sigma_{I}$ associated with a given rainfall rate class: $\left\langle\mu_{I}\right\rangle \propto<\sigma_{I}>$ where the symbol $<\cdots\rangle$ indicates the rainfall rate class average.

Hereby, we show that given a fixed range of values for the mean diameter $\mu_{I}$, the more negative (positive) is the skewness class to which the renormalization time interval belongs the smaller (larger) is the standard deviation $\sigma_{I}$ of the drop diameters. As for the result depicted in Fig. 3, we divide the entire Darwin dataset in subsets according to the skewness class. Then we fix a range of values for the mean diameter $\mu_{I}$ and for each subset we evaluate the median of the observed values of $\sigma_{I}$ when $\mu_{I}$ is in the given range. The results are reported in Fig. 5. The vertical lines depict the range of $\mu_{I}$ values considered. The median of the $\sigma_{I}$ values is calculated only when at least 10 time intervals are in the range considered. We see how the median value of $\sigma_{I}$ increases as the skewness class goes from -2 to +2 .

\section{Conclusions}

"Averaged" renormalized spectra relative to stratiform and convective precipitation at Darwin (AU) possess a common shape (Ignaccolo and De Michele, 2010). Is this result the consequence of a more general invariance: the invariance of single instantaneous renormalized spectra? Here, we have investigated this possibility providing a positive answer extensively. Since instantaneous renormalized spectra have all zero mean and unit variance, the skewness was taken as a parameter to test invariance. The probability $\operatorname{Pr}\left(\gamma_{I}\right)$ of skewness values is peaked around the values 0.64 . The dispersion of skewness values around the most probable one is quantified in terms of skewness classes. The skewness class $\mathrm{s} 0$ centered around the most probable value contains $\sim 57 \%$ of the

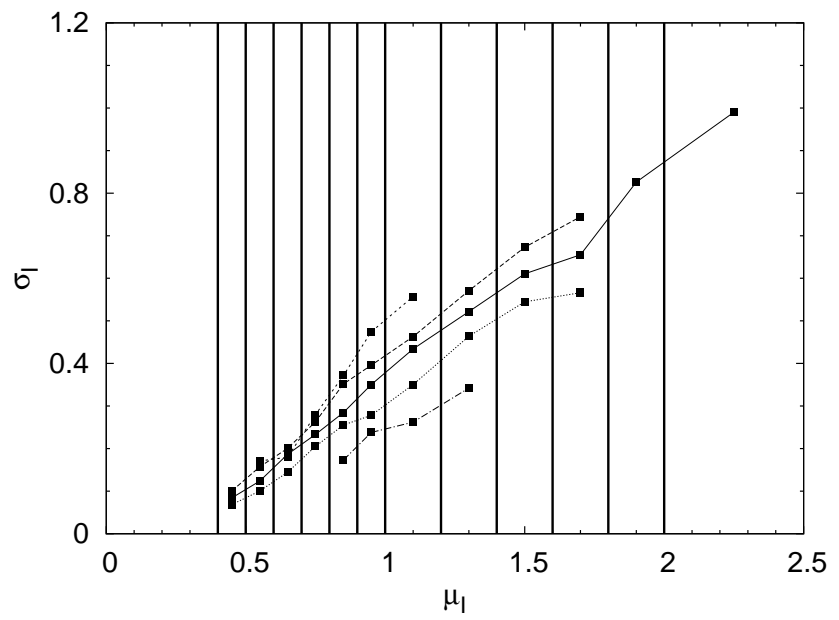

Fig. 5. The median value of the parameter $\sigma_{I}$ for a given range of $\mu_{I}$. The results relative to five different skewness classes are shown: s0 (solid line), s+1 (long-dashed line), s +2 (short-dashed line), $s-1$ (dotted line), and $s-2$ (dotted-dashed line). Vertical lines indicate the different ranges of values of $\mu_{I}$ used to calculate the median.

renormalization time intervals corresponding to $\sim 60 \%$ of the total drops in the database. The remaining renormalization time intervals essentially belong to the skewness classes $\mathrm{s}+1$ and $\mathrm{s}-1$ with only $\sim 2 \%$ of intervals in the skewness classes associated with the tails of the probability $\operatorname{Pr}\left(\gamma_{I}\right)$. The results of this classification allow us (using Eq. 7) to define the most common renormalized spectra $S\left(D_{R}\right)$ as the density of renormalized drop diameters $D_{R}$ relative to the subsets of renormalization time intervals inside the skewness class $\mathrm{s} 0$. We use the most common distribution to produce an artificial database and prove that part of the observed dispersion of the skewness around the most probable value is due to sampling limitations since instantaneous spectra are derived from 1 min drop counts (Fig. 4). About $8 \%$ of the renormalization time intervals of the artificial database belong to the skewness classes $\mathrm{s}+1$ and $\mathrm{s}-1$ compared to $\sim 40 \%$ for the real data. In this sense one can estimate the sampling effects to be responsible for approximatively one fifth $(8 / 40)$ of the observed dispersion of skewness values.

Another issue we have addressed here, is how the skewness of renormalized instantaneous spectra depends on the two renormalization parameters: mean and standard deviation of drop diameters. For a given range of mean diameter values, we have calculated the median of the observed values of the standard deviation for each skewness class. We have found that the median increases as the skewness class goes from -2 to +2 . That is given a value of the mean diameter, larger (smaller) values of the skewness are associated with large (small) values of the standard deviation.

The probability densities $p_{G}\left(D_{R}\right)$ for the databases of a given skewness class are independent from the stratiform versus convective classification which is not a result expected 
a priori: stratiform and convective instantaneous spectra with the "same" skewness value have the "same" renormalized spectra. More in general the adoption of the skewness as metric to measure the equality of renormalized spectra can be useful in comparing instantaneous renormalized spectra at different locations with different meteorological regimes. E.g. in the companion paper (Ignaccolo and De Michele, 2012) we will discuss the properties of instantaneous renormalized spectra in presence of orography. Stratiform, convective and orographic are the three main kinds of precipitation.

Acknowledgements. We are thankful to C. R. Williams and the National Oceanic and Atmospheric Administration for the public availability of the data set. M. I. thankfully acknowledges the support of Accademia Nazionale dei Lincei (Italy) through "B. Segre" scholarship. C. D. M. thankfully acknowledges Comune di Milano (Italy) through BIODESCESA project.

Edited by: R. Uijlenhoet

\section{References}

Campos,E. F., Zawadzki, I., Petitdidier, M., and Fernandez, W.: Measurement of raindrop size distributions in tropical rain at Costa Rica, J. Hydrol., 328, 98-109, 2006.

Feingold, G. and Levin, Z.: Application of the lognormal raindrop distribution to differential reflectivity radar measurements (ZDR), J. Atmos. Ocean. Tech., 4, 377-382, 1987.

Hazenberg, P., Nan, Y., Boudevillain, B., Delrieu, G., and Uijlenhoet, R.: Scaling of raindrop size distributions and classification of radar reflectivity - rain rate relations in intense Mediterranean precipitation, J. Hydrol., 402, 179-192, 2011.

Ignaccolo, M. and De Michele, C.: Statistical collapse of stratiform and convective drop diameter distributions at the ground, Geophys. Res. Lett., 37, L24402, doi:10.1029/2010GL045454, 2010.

Ignaccolo, M. and De Michele, C.: Skewness as measure of the invariance of instantaneous renormalized drop diameter distributions - Part 2: Orographic precipitation, Hydrol. Earth Syst. Sci., 16, 329-343, doi:10.5194/hess-16-329-2012, 2012.
Ignaccolo, M., De Michele, C., and Bianco, S.: The drop-like nature of rain and its invariant statistical properties, J. Hydrometeorol., 10, 79-95, 2009.

Joss, J. and Gori, E. G.: Shapes of raindrop size distributions, J. Appl. Meteorol., 17, 1054-1061, 1978.

Konstinski, A. B. and Jameson, A. R.: Fluctuation properties of precipitation, Part I: On Deviations of Single-Size Drop Counts from the Poisson Distribution, J. Atmos. Sci., 54, 2174-2186, 1997.

Maki, M., Keenan, T. D., Sasaki, Y., and Nakamura, K.: Characteristics of the raindrop size distribution in tropical continental squall lines observed in Darwin, Australia, J. Appl. Meteorol., 40, 1393-1412, 2001.

Marshall, J. S. and Palmer, W.: The distribution of raindrops with size, J. Meteorol., 5, 165-166, 1948.

Sauvageot, H. and Lacaux, J. P.: The Shape of Averaged Drop Size Distributions, J. Atmos. Sci., 52, 1070-1083, 1995.

Sekhon, R. S. and Srivastava, R. C.: Doppler radar observations of drop-size distributions in a thunderstorm, J. Atmos. Sci., 28, 983-994, 1971.

Sempere Torres, D., Porrà, J. M., and Creutin, J. D.: A general formulation for raindrop size distribution, J. Appl. Meteorol., 33, 1494-1502, 1994.

Smith, J. A.: Marked point process models of raindrop-size distributions, J. Appl. Meteorol., 32, 284-296, 1993.

Testud, J., Oury, S., Black, R. A., Amayenc, P., and Dou, X.: The concept of normalized distribution to describe raindrop spectra: a tool for cloud physics and cloud remote sensing, J. Appl. Meteorol., 40, 1118-1140, 2001.

Tokay, A. and Short, D. A.: Evidence from tropical raindrop spectra of the origin of rain from stratiform versus convective clouds, J. Appl. Meteorol., 35, 355-371, 1996.

Uijlenhoet, R., Smith, J. A., and Steiner, M.: The microphysical structure of extreme precipitation as inferred from ground-based raindrop spectra, J. Atmos. Sci., 60, 1220-1238, 2003.

Ulbrich, C. W.: Natural variations in the analytical form of the raindrop size distribution, J. Clim. Appl. Meteorol., 22, 1764-1775, 1983.

Willis, P. T.: Functional fits to some observed dropsize distributions and parameterization of rain, J. Atmos. Sci., 41, 1648-1661, 1984. 